\title{
Rapid rotation of a sunspot associated with flares
}

\author{
X. L. Yan ${ }^{1,2}$ and Z. Q. Qu ${ }^{1}$ \\ 1 Yunnan Astronomical Observatory/National Astronomical Observatories, Chinese Academy of Sciences, Kunming, \\ Yunnan 650011, PR China \\ e-mail: yanxl@ynao.ac.cn \\ 2 Graduate School of Chinese Academy of Sciences, Zhongguancun, Beijing, PR China
}

Received 8 January 2007 / Accepted 3 March 2007

\section{ABSTRACT}

\begin{abstract}
Context. Active region NOAA 10424 observed on August 5, 2003 is studied in detail by using TRACE, SOHO/MDI, BBSO H $\alpha$ monograph, and GOES data. This investigation focuses on the sunspot rotation and its relation with the eruptive phenomena by analyzing the magnetic configuration that the rotation results in.

Aims. It is shown that there is a close relationship between the sunspot rotation and the emerging kinked magnetic $\Omega$-loops, where the flares occur.

Methods. Through tracing the traceable features motion by using the TRACE white-light images, one can get the rotation velocities of the umbra, the penumbra, the area near the penumbra, and the area far from the penumbra. Furthermore, the evolution of the emerging kinked magnetic $\Omega$-loops and magnetic fields were studied.

Results. For the sunspot with positive polarity, the umbra, the penumbra, and the area near the penumbra exhibit a conspicuous counterclockwise rotation. Moreover, the velocities decrease from the umbra through the penumbra to the area near the penumbra. It is interesting that the rotation of the umbra, the penumbra, and the area near the penumbra are opposite to that of the area far from penumbra. The rotation velocities of the umbra, the penumbra, polarity separation, and total magnetic flux increase with time. During the largest event (M1.7/Sn flare), emerging kinked magnetic $\Omega$-loops are observed from TRACE $171 \AA$ images.

Conclusions. The different rotation speeds of the different parts of the sunspot cause twist, and then the twist is injected through the chromosphere into the corona to trigger the flares.
\end{abstract}

Key words. Sun: sunspots - Sun: flares - Sun: magnetic fields

\section{Introduction}

It is well known that a solar flare is a sudden release of energy that was previously stored in the magnetic field, which is a process of rapid transformation of the magnetic energy of the active region into the kinetic energy of particles, radiation, plasma flows and heat. It is now widely accepted that flares derive their power from the free energy stored in stressed or non-potential magnetic fields in the active regions (Zirin et al. 1973; Hagyard et al. 1984). However, how the magnetic energy is stored and released still has not been proved well enough, thus it remains a very important issue in solar physics.

Although authors have investigated the relationship between the shear angle variation and the occurrence of flares, there are many contradictory speculations on this relationship. For instance, after the appearance of a flare, the shear angle may decrease (Rausaria et al. 1993), stay unchanged (Chen et al. 1994), or even increase (Wang et al. 1993, 1994). On the other hand, other authors have studied the correlation between the variations of the photospheric magnetic field and flares. Kosovichev \& Zharkova (1999) report that there is a sudden decrease in the magnetic energy after the flare. But Spirock et al. (2002) observed a significant increase in the magnetic field; Furthermore, investigators have observed the rotational motions of sunspots (Ding et al. 1987; Khutsishvili et al. 1998; Raman et al. 1998, 2006; Gerrard et al. 2002; Brown et al. 2002, 2003; Williams et al. 2004; Gopasyuk et al. 2005; Tian et al. 2005, 2006), and some of them related this to the occurrence of the eruptive phenomena.

But how is the magnetic energy stored in a non-potential configuration? Zhao \& Kosovichev (2003) have found subsurface horizontal vortical flows under NOAA 9114. The rotation of a sunspot in the photosphere may cause the injection of twist into the corona, proven by the TRACE EUV images and by the S-shaped or inverse S-shaped structures observed in soft X-ray by Yohkoh/SXT (Pevtsov 2002). Photospheric motions are considered as either pre-flare phenomena or resources of the magnetic energy accumulation, so they are utilized to investigate the flare. In fact, the rotation motion of separate and individual sunspots and groups of sunspots is a very frequent phenomenon. However, it is important that the rotation of magnetic foot-points in the photosphere can result in twists to be injected through the chromosphere into the corona, and these twisted structures are likely to erupt as flares (Brown et al. 2002) or/and coronal mass ejections (CMEs) (Gibson et al. 2004). Brown et al. (2002, 2003) calculated the rotation of sunspot by using a kind of geometric technique and find that the rotation of the umbra is slower than that of the penumbra. As the umbra contains less features than the penumbra in general, this makes the measurement of umbral rotation less reliable than for the penumbral.

Nevertheless, to our knowledge, rapid rotation of the umbra of sunspots about their centers has been observed very rarely. In this paper, we present an example of rapid rotation of the umbra, the penumbra, the area near the penumbra, and the area far from 
the penumbra about the sunspot center by using a new kind of geometric technique different from the one used in Brown et al. (2002, 2003). This study will add one significant and convinced proof that the rotation motions trigger the flares.

\section{Observations and data reduction}

NOAA 10424 was the largest and most active region during its appearance on the disk. The largest event of the period, an M1.7/Sn flare, was observed by GOES at 12:49 UT on August 5. This flare was associated with a weak type II radio sweep measured as $339 \mathrm{~km} \mathrm{~s}^{-1}$. The magnetic field structure developed into a beta-gamma-delta configuration on August 6 and its growth phase ended and a steady decay phase arrived on August 7.

We analyze the physical process by using white light $(5000 \AA)$ and Fe IX/X $171 \AA$ images of high-resolution and high-cadence from the Transition Region and Coronal Explorer (TRACE) (Handy et al. 1999). Other data include full disk magnetograms by MDI (SOHO/MDI $96 \mathrm{~m}$ line-of-sight magnetograms) on board the Solar and Heliospheric Observatory (SOHO; Domingo et al. 1995), $\mathrm{H} \alpha$ images from Big Bear Observatory (BBSO), and X-Ray plot by YOHKOH SXT (Tsuneta et al. 1991).

From the TRACE white-light images $\left(0.5\right.$ pixel $\left.^{-1}\right)$ with $1^{\prime \prime}$ spatial resolution and a temporal resolution of 1.5-2.5 minutes, one can easily calculate the rotation angle, polarity separation, and tilt angle precisely by using a form of the geometric method introduced below. As is well-known, the rapid evolution of the magnetic loops generally signifies that of the coronal magnetic field. Otherwise, the coronal images obtained by TRACE $171 \AA$ are used to investigate the change of magnetic loops in the coronal environment. The data of the space-board instruments are disposed by the standard Solar Software (SSW) codes before further analysis. Using these data, we can trace the evolution of sunspot umbra rotation and magnetic flux loops conveniently.

\section{The rotation of the sunspot}

As the umbra has low image intensity compared with the penumbra or the Quiet Sun region, we define the lowest intensity point as the center of the sunspot umbra "*" for the negative polarity. For the sunspot with positive polarity, we take the intersection ("o") of the two light bridges as the center of the sunspot umbra. In order to measure the rotation angle, it is necessary to define a coordinate system. The rotation axis of the sun is adopted as $Y$-axis, and the $X$-axis is drawn perpendicular to the $Y$-axis. These axes are drawn on TRACE WL images (see Fig. 1), while the point " " is selected as the intersection of these coordinate axes. The rotation angle $(\alpha)$ of the umbra refers to the counterclockwise angle between the $Y$-axis and the line along the larger light bridge in the umbra. The angle between the line passing through the centers of the two sunspot umbrae and the $X$-axis is defined as tilt angle $(\beta)$. This angle indicates the relative motion of the two sunspots. "C", "B", and "A" are samples signifying the points in the penumbra, in the area near the penumbra and in the area far from the penumbra respectively. As the positions of the three points can be traced clearly, one can calculate their rotation angle (between the positive direction of the $X$-axis and the line connecting "A" or "B" or " $C$ " and the center of the umbra). Any time variation of the angles gives the change in the orientation of the umbra, the penumbra, the area near the penumbra, and the area far from the penumbra, thus it indicates the rotational motion. This method is specific and convenient for calculating the

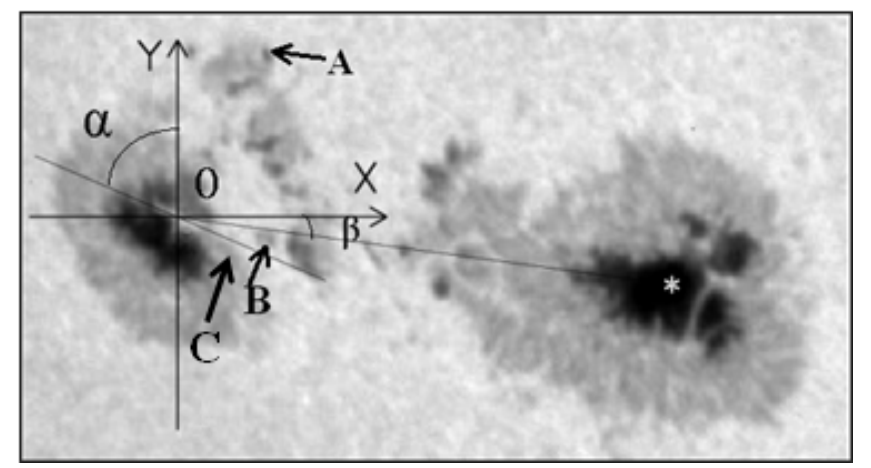

Fig. 1. Calculation of the sunspot rotation. The rotation axis of the sun is defined as $Y$-axis, while $X$-axis is drawn perpendicular to the $Y$ axis. The rotation angle $(\alpha)$ is calculated counterclockwise between the $Y$-axis and the line along the largest light bridge in the umbra. The white "*" and "o" on the image are the centers of the two sunspots umbrae. The tilt angle $(\beta)$ is defined as the angle between the line connecting the centers of the two sunspot umbrae and the $X$-axis. "C", "B", and "A" are the signifying points in the penumbra, in the area near the penumbra and in the area far from the penumbra, respectively.

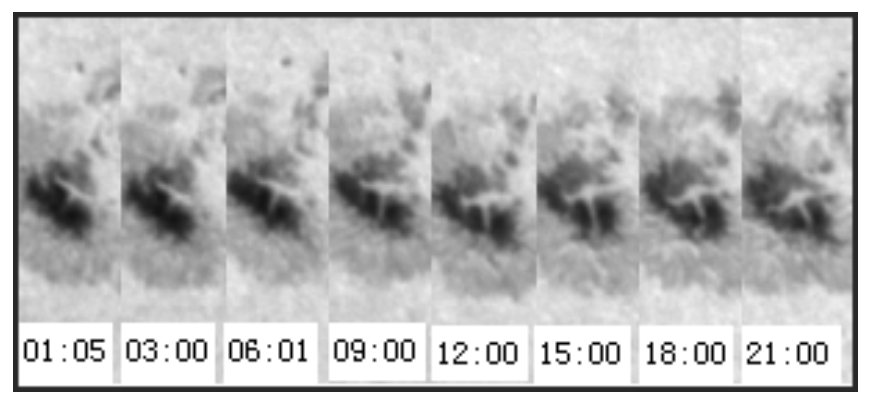

Fig. 2. A sequence of the white-light images stacked in increasing phase on August 5, 2003. The rotation of light bridges can be seen clearly.

rotation in this specific case, and it is different from the method that measures the magnetic shear from the vector magnetograms. Finally, the length of the straight line segment between the centers of the two sunspot umbrae is taken as the measure of the polarity separation. All the parameters defined are indicated in Fig. 1.

The sunspot with positive polarity has two light bridges. From WL images, the counterclockwise umbral rotation around its center is conspicuous (Fig. 2). By the method introduced above, we analyze TRACE WL images to identify the morphological changes. From Fig. 3a, we can see the rotation angle $(\alpha)$ increasing by $64^{\circ}$ in one day. The average rotation speed is $2.7^{\circ} h^{-1}$. This is almost the same as the penumbral rotation velocity obtained by Brown et al. (2002, 2003). In fact, it is found that the rotation speed decreases with time in this case. Meanwhile, the rotation angle of the penumbra and the area near the penumbra increases slowly at first and then decreases a little (see Figs. 3b, c). In contrast, the rotation angle of the area far from the penumbra decreases clockwise from $65^{\circ}$ to $38^{\circ}$ (see Fig. 3d). It is astonishing, that, according to the calculations, the rotation velocities of the penumbra, the area near the penumbra, and the area far from the penumbra are smaller than that of the umbra. Moreover, the velocities become lower from the umbra to the area near the penumbra. It is worth noting that the change in the rotation angle is much greater than the differential rotation (Brown et al. 2003) and that there is a small magnitude of back-rotation of the sunspot. This may be ascribed to torsional oscillations (Williams et al. 2004). 

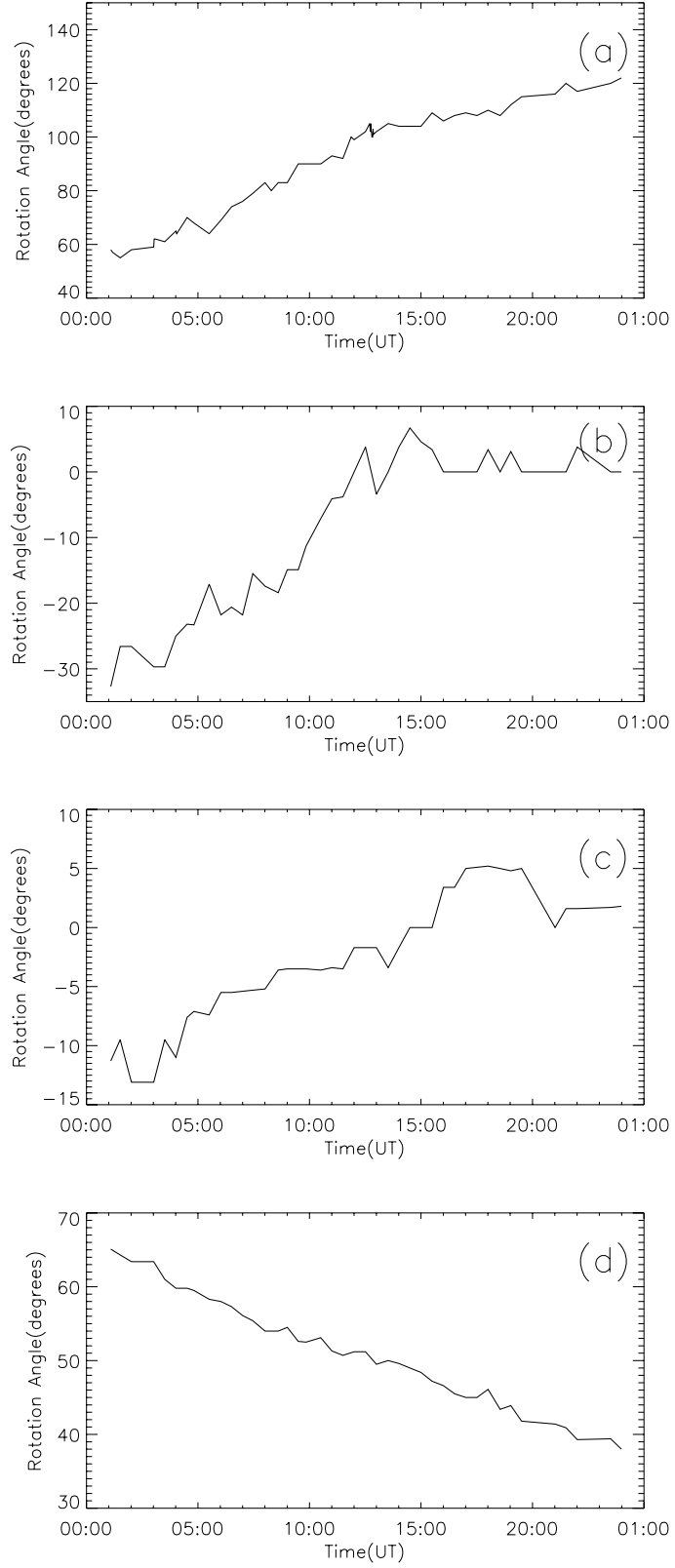

Fig. 3. Plots showing the change of rotation angle with time on August 5, 2003. The plots of a), b), c) and d) indicate the change of the rotation angle of the umbra, the penumbra, the area near the penumbra and the area far from the penumbra respectively.

Not only does the sunspot rotation become the main feature of the active region, but five flares also occurred that day accompanying the complicated movements. Therefore, we conclude that there is a close relation between the flares and the rotation of the sunspot. Otherwise, the polarity separation increases with time (see the lower panel of Fig. 4). One can find that the magnitude of the tilt angle (see the upper panel of Fig. 4) decreases before the occurrence of M1.7 flare.

\section{Flares}

Flares were seen clearly in this region from GOES 10 on August 5, 2003 (see Fig. 5). There were five flares occurring
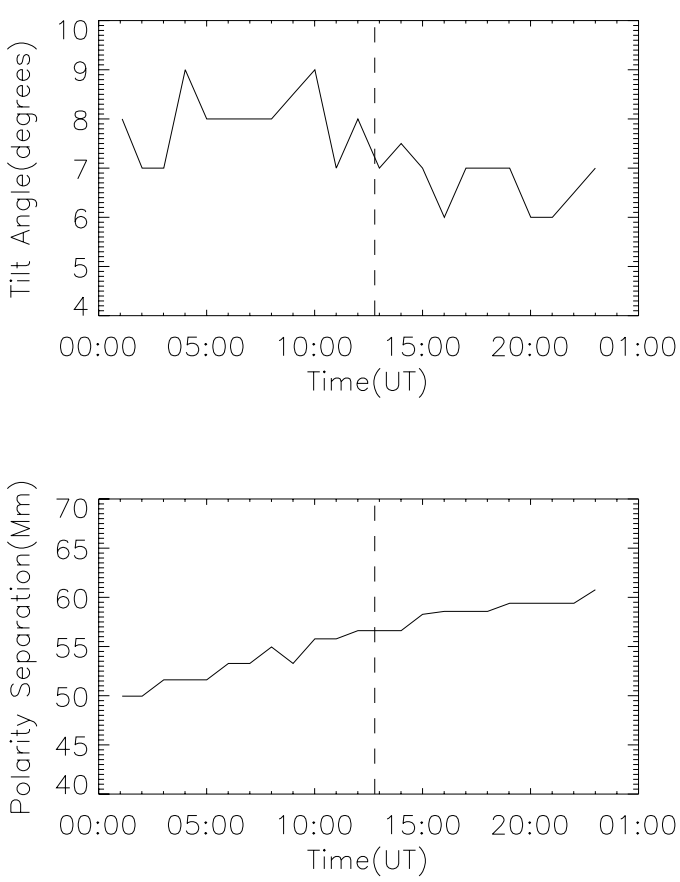

Fig. 4. The upper panel gives change of the tilt angle with time. The lower one shows the polarity separation variation with time. The dashed line denotes the beginning time of M1.7 flare.

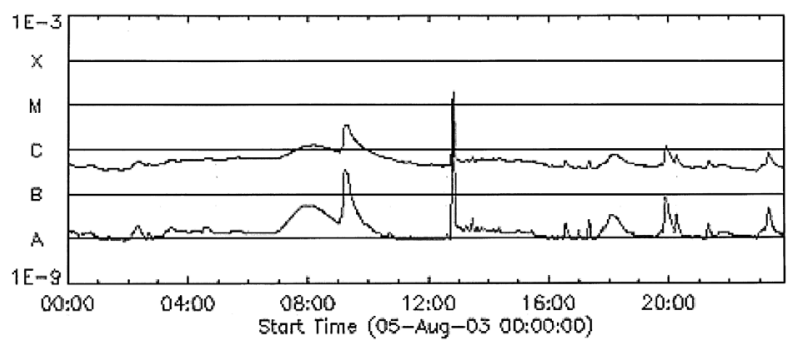

Fig. 5. GOES10 plot showing X-ray emission during the rotation of the sunspot with positive polarity on August 5, 2003.

in this active region that day (listed in Table 1). The largest flare (M1.7) started at 12:43 UT and peaked at 12:49 UT.

\subsection{The M1.7 flare}

Figure 5 presents the soft X-ray emission of the largest flare from GOES 10. It lasted $15 \mathrm{~min}$ from 12:43 UT to 12:58 UT and reached its peak at 12:49 UT. During the flare, the amplitude of the tilt angle became smaller. This indicates that the magnetic tension force became stronger between the leading and subsequent sunspots. Figure 6a depicts the flare reaching its optical emission peak by the WL image, while the two bright patches are positioned by the arrows (see Fig. 6b) in the difference image, and the two foot-points of the magnetic loops located in different magnetic polarities (see Fig. 6c). From TRACE $171 \AA$ images (see Fig. 7a), the twisted flux ropes emerging can be found easily. Afterwards, the twisted flux ropes expanded rapidly and formed kinked magnetic flux-loops (see the box in Fig. 7b). Furthermore, two larger $\Omega$-loops interacted each other. In the following process, two new thin magnetic loops were formed swiftly (see Fig. 7c) after the two larger kinked $\Omega$-loops turned 
Table 1. The flare list.

\begin{tabular}{crrrrc}
\hline \hline Number & Start(UT) & Peak (UT) & End(UT) & Class(GOES) & Location \\
\hline 1 & $12: 43$ & $12: 49$ & $12: 51$ & M1.7 & S16E33 \\
2 & $17: 17$ & $17: 21$ & $17: 23$ & B5.9 & $?$ \\
3 & $19: 47$ & $19: 55$ & $20: 02$ & C1.1 & S15E29 \\
4 & $20: 12$ & $20: 16$ & $20: 19$ & B7.1 & $?$ \\
5 & $23: 13$ & $23: 18$ & $23: 24$ & B8.5 & $?$ \\
\hline
\end{tabular}

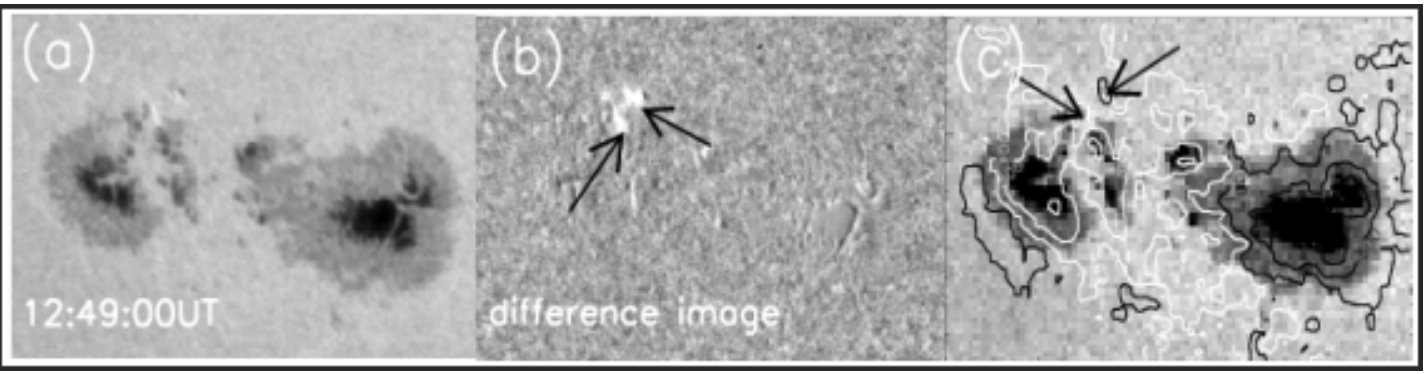

Fig. 6. a) TRACE white-light image at 12:49:00 UT. b) the difference image (12:49:00 UT-12:46:00 UT). The two arrows point to two bright patches. c) TRACE white-light image at 12:49:00 UT with SOHO/MDI longitudinal magnetic field superposed. The two arrows point to the same area as b). The white/black contours represent positive/negative longitudinal magnetic fields. The magnetic contour levels are 100, 500, 1000, 1500 , and $2000 \mathrm{G}$. The field of view (FOV) of a)-c) is $160^{\prime \prime} \times 110^{\prime \prime}$.
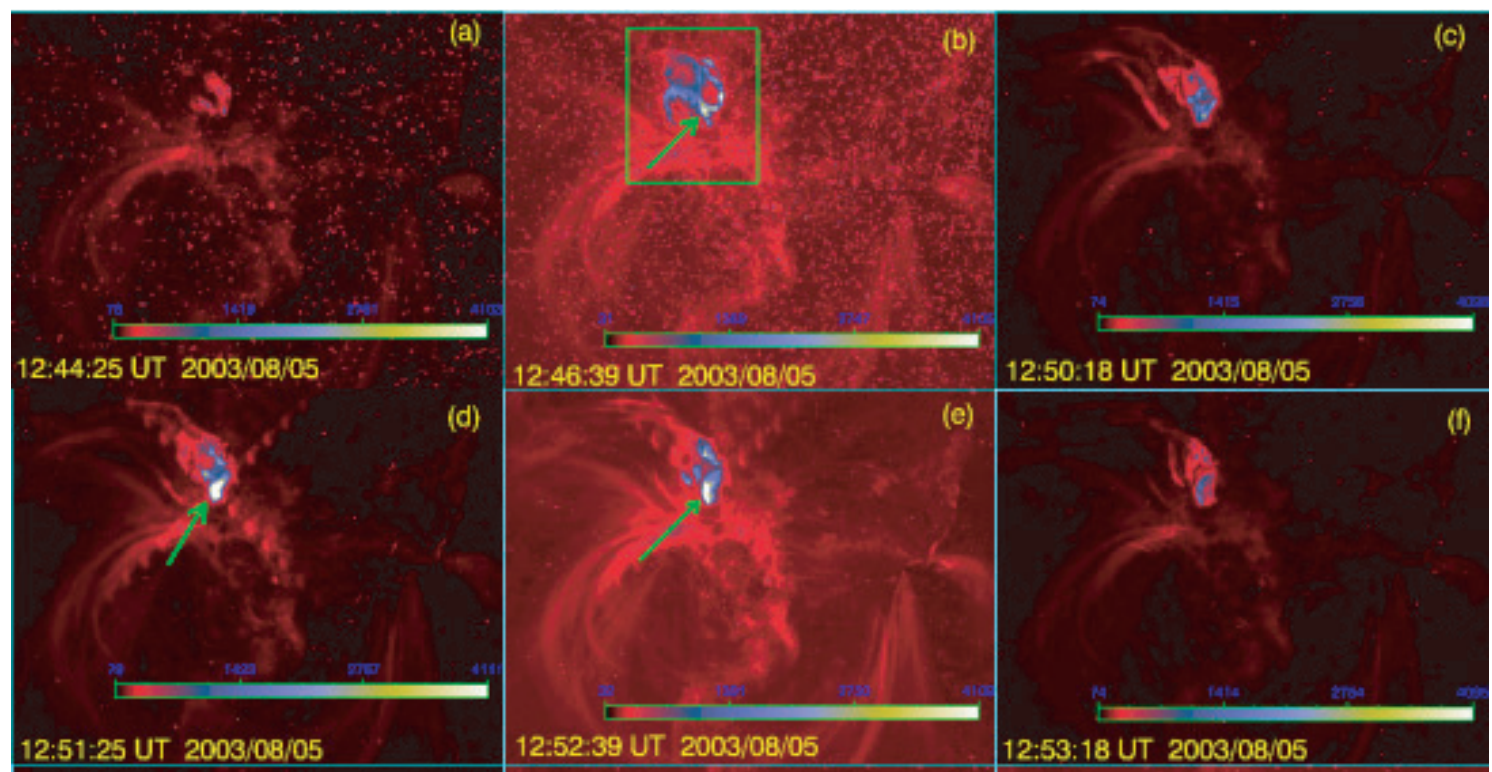

12:50:18 UT 2003/08/05
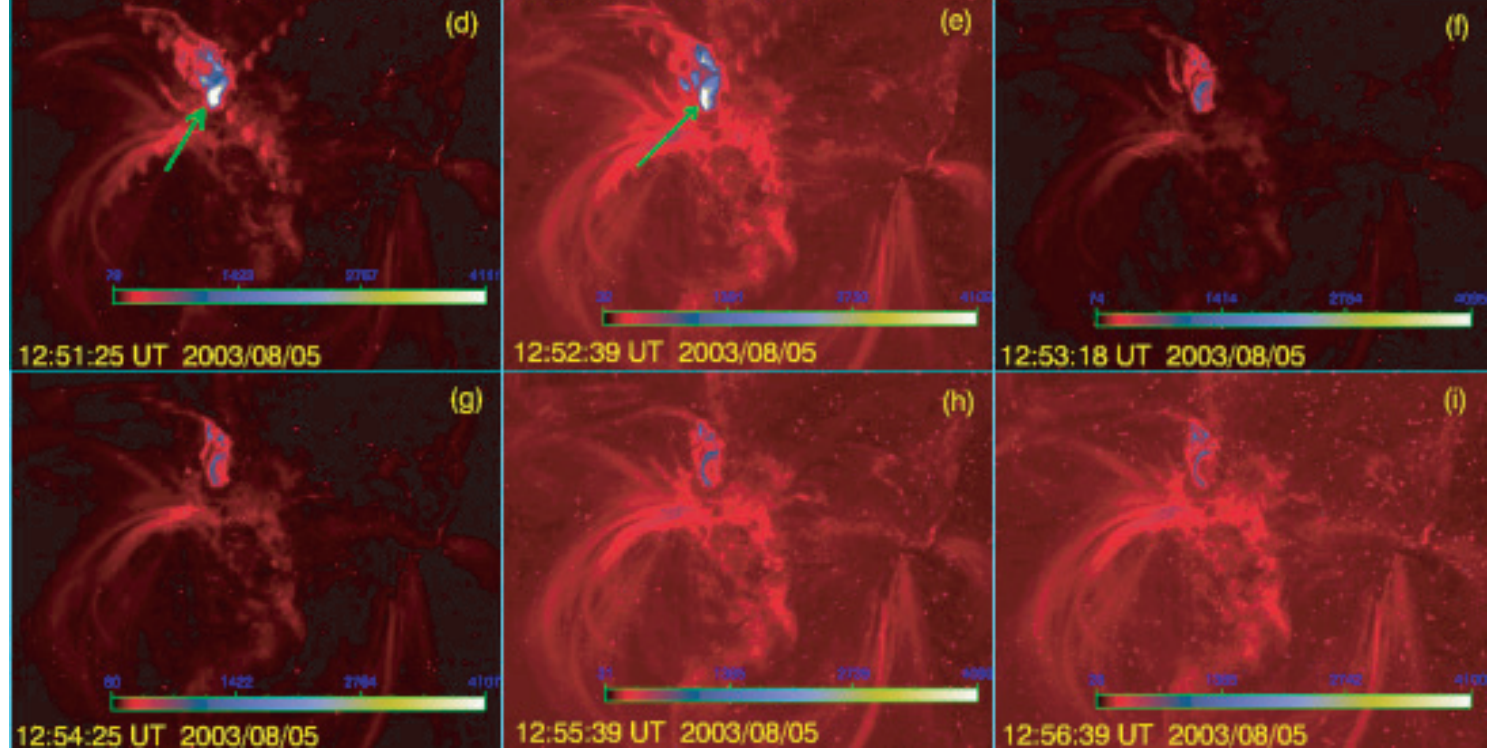

(h)

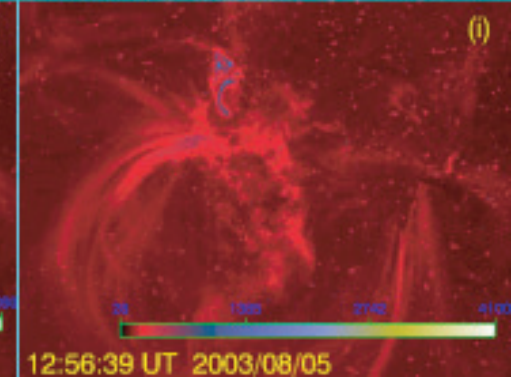

Fig. 7. A sequence of TRACE images acquired at $171 \AA$, showing the evolution of magnetic flux loops in the corona during the occurrence of the M1.7 flare. FOV: $200^{\prime \prime} \times 150^{\prime \prime}$. The color bars indicate the intensity of the image.

into small ones. They were open in turn (Figs. 7d, e). From these phenomena, one can naturally deduce that the two magnetic loops reached kink instability and then eruption occurred. After the eruption, the post flare loops emerged. Finally, the loop shrank and disappeared (see Figs. 7f-i). During the change in configuration of the loops, the intersection of the two forward larger loops, was markedly brighter than the surrounding area. It remained bright from 12:51:25 UT to 12:58:13 UT. This implies the magnetic reconnection occurred.

From the above descriptions, the causal chain emerges clearly: the rotation of the sunspot with positive polarity caused the loops to be twisted in the photosphere and then the twist 


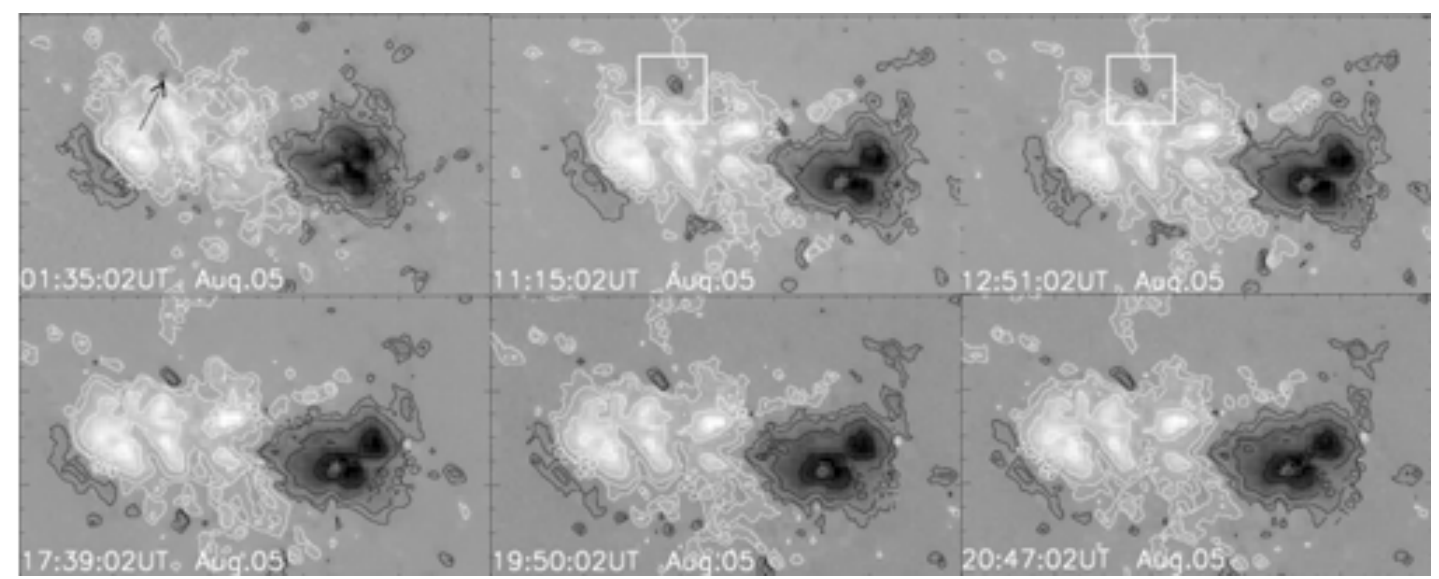

Fig. 8. A sequence of SOHO/MDI 96 m line-of-sight magnetograms observed 2003 August 5 . Fov : $200^{\prime \prime} \times 120^{\prime \prime}$. White (black) patches signify positive (negative) longitudinal magnetic fields. The contour levels of the longitudinal field indicate 100, 200, 500, 1000, 1500, 2000 G. One foot of the M1.7 flare loop is designated by the arrow or the boxes.

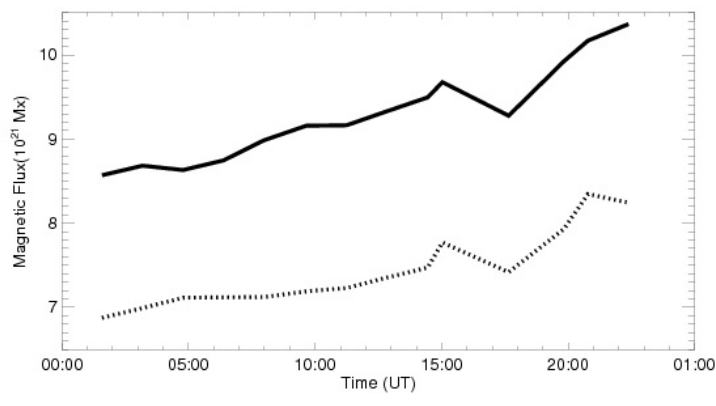

Fig. 9. Evolution of the line-of-sight magnetic flux in AR 10424 on 2003 August 5. The solid line represents the following flux (positive), while the dashed line represents the leading flux (negative).

turned into the corona. The M1.7 flare could be ascribed to the interaction of the kinked $\Omega$-loops and then to the kink instability. It is worth pointing out that Gibson et al. (2002, 2004) observed magnetic flux ropes in the corona and simulated the rotation of the sunspot. And recently, Inoue et al. (2006) did a prosecuted three-dimensional simulation of the dynamic flux rope in the solar corona. According to the above discussion, the rotation of the sunspot and the resulting twisted magnetic flux loops are found in the corona. From the movie (http : / /hea-www.harvard.edu/trace/flare_catalog/ 2003.html), one can also clearly see the process of the M1.7 flare.

\subsection{The evolution of the whole active region}

From Fig. 2, as pointed out above, one can find that not only the sunspot with positive polarity rotated and the rotation speed experienced variation that day, but the polarity separation also increased slowly with time. From a time series of 96-min cadence magnetograms, one can also see the evolution of the magnetic field of the whole active region on August 5, 2003 (see Fig. 8). The arrow and the boxes point to a negative polarity pore that is one foot-point of the M1.7 flare loop in the photosphere. Using the magnetograms, we calculated the change in the magnetic flux (see Fig. 9) and find that both positive and negative magnetic flux increased on the whole. And it is interesting that the increase and the decrease in the two polarities are almost in cadence, which signifies that they belong to the same emerging

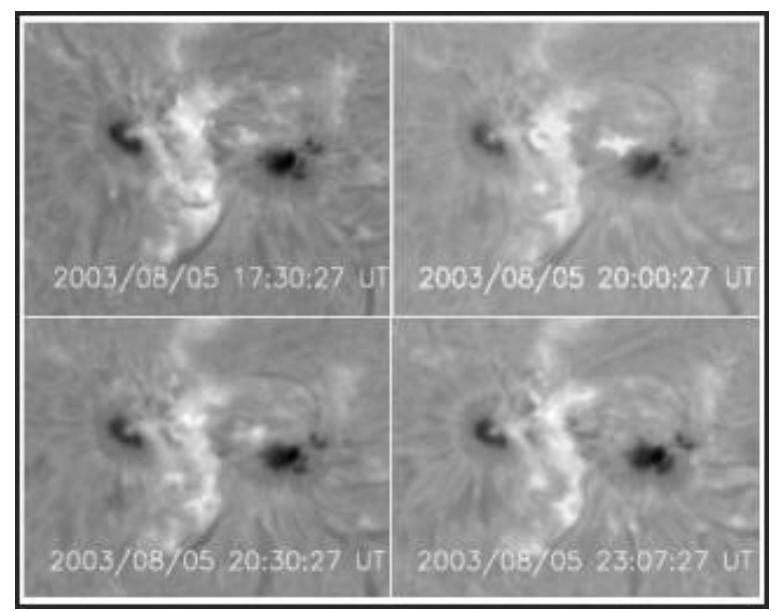

Fig. 10. BBSO $\mathrm{H} \alpha$ images. The bright patches indicate flares in AR10424. FOV: $210^{\prime \prime} \times 158^{\prime \prime}$.

loop system. From Fig. 8, one can see the negative pore occupied a very small area at the beginning and then increased with time in the following. This flux change did not lead just to the M1.7 flare, from BBSO $\mathrm{H} \alpha$ images (see Fig. 10), the other flares close to the neutral line can be seen and the largest patches of different flares have the same position and similar shapes.

\section{Summary and discussion}

In the above sections, we have investigated the active region NOAA 10424 on August 5, 2003 and obtained the following findings:

1. For the sunspot with positive polarity, the umbra, the penumbra, and the area near the penumbra exhibit conspicuous counterclockwise rotation but the area far from the penumbra rotates in the opposite direction in this region.

2. The rotation velocities of the penumbra, the area near the penumbra, and the area far from the penumbra are smaller than that of the umbra. Moreover, the velocities decrease from the umbra through the penumbra to the area near the penumbra.

3. Polarity separation and total magnetic flux of the whole active region increased with time during the flare occurrence. 
4. The kinked magnetic $\Omega$-loops are identified, and their interaction in the corona can be seen very clearly. The intersection of the kinked magnetic $\Omega$-loops is brighter than its surrounding, where magnetic reconnection could happen.

Findings 1 and 4 provide direct evidence that photospheric motion makes magnetic loops kinked through the photosphere and chromosphere into corona, and then flares are caused. As is well known, the Coriolis force can make sunspots rotate in a clockwise (counterclockwise) direction in the northern (southern) while differential rotation gives rise to the reverse effect. Cannon \& Marquette (1991) and Marquette \& Martin (1988) found in their studies on sunspot rotation that the origin of the rotation is due neither to the emergence of new flux nor to the interaction with the surrounding field. Bao et al. (2002) analyzed several origins of twist of magnetic flux tubes. However, many different viewpoints exist in this field. For example, Brown et al. (2003) think the differential rotation does not play a major role in producing sunspot rotation.

As the umbra and the penumbra form at different depths, we suggest that the counterclockwise rotation of the umbra and the penumbra is caused by the Coriolis force and the rotation of the outer penumbra is caused by differential rotation. Both the rotation of the umbra, the penumbra, the area near the penumbra and the area far from penumbra cause twist to be injected through the chromosphere into the corona, and these twisted flux tubes store sufficient magnetic energy to trigger the flares.

Acknowledgements. The authors thank the SOHO/MDI, TRACE, BBSO, and YOHKOH SXT consortia for their data. This work is supported by the National Science Foundation of China (NSFC) under the grant number 10673031 and National Basic Development Project 973 under the grant number $2006 \mathrm{CB} 806300$.

\section{References}

Bao, S. D., Sakurai, T., \& Suematsu, Y. 2002, ApJ, 573, 445

Brown, D. S., Nightingale, R. W., Alexander, D., et al. 2002, Proceedings of the Magnetic Coupling of the Solar Atmosphere Euroconference and IAU Coll. 188, ed. H. Sawaya-Lacoste, ESA SP-505 (ESA Publications Division), 261 Brown, D. S., Nightingale, R. W., Alexander, D., et al. 2003, Sol. Phys., 216, 79 Chen, J. M., Wang, H. M., Zirin, H., et al. 1994, Sol. Phys., 154, 261 Cannon, A. T., \& Marquette, W. H. 1991, Sol. Phys., 131, 69

Ding, Y. J., Hong, Q. F., Wang, H. Z., et al. 1987, Sol. Phys., 107, 221 Domingo, V., Fleck, B., \& Poland, A. I. 1995, Sol. Phys., 162, 1

Gerrard, C. L., Brown, D. S., Mellor, C., et al. 2003, Sol. Phys., 213, 39

Gibson, S. E., Low, B. C., Leka, K. D., et al. 2002, Proceedings of the Magnetic Coupling of the Solar Atmosphere Euroconference and IAU Coll. 188, ed. H. Sawaya-Lacoste, ESA SP-505 (ESA Publications Division), 265

Gibson, S. E., Fan, Y., Mandrini, C., et al. 2004, ApJ, 617, 600

Gopasyuk, S. I., \& Gopasyuk, O. S. 2005, Sol. Phys., 231, 11

Hagyard, M. J., Teuber, D., West, E. A., et al. 1984, Sol. Phys., 91, 115

Handy, B. N., Acton, L. W., Kankelborg, C. C., et al. 1999, Sol. Phys., 187, 229 Inoue, S., \& Kusano, K. 2006, ApJ, 645, 742

Khutsishvili, E., Kvernadze, T., \& Sikharulidze, M. 1998, Sol. Phys., 178, 271

Kosovichev, A. G., \& Zharkova, V. V. 1999, Sol. Phys., 190, 459

Marquette, W. H., \& Martin, S. F. 1988, Sol. Phys., 117, 227

Pevtsov, A. A. 2002, Sol. Phys., 207, 111

Rausaria, R. R., Sundara, R. K., Aleem, P. S. M., et al. 1993, Sol. Phys., 146, 137

Sundara Raman, K. 2006, Proceedings of Preparation of Camera-Ready Papers for ILWS Workshop, GOA, february 19-20, 2006

Sundara Raman, K., Selvendran, R., \& Thiagarajan, R. 1998, Sol. Phys., 180, 331

Spirock, T., Yurchyshyn, V., \& Wang, H. M. 2002, ApJ, 572, 1072

Tsuneta, S., Acton, L., Bruner, M., et al., et al. 1991, Sol. Phys., 136, 37

Tian, L., \& Alexander, D. 2006, Sol. Phys., 233, 29

Tian, L., Liu, Y., Yang, J., et al. 2005, Sol. Phys., 229, 237

Wang, H., \& Tang, F. 1993, ApJ, 407, 89

Wang, H., Ewell, M. W., Zirin, H., et al. 1994, ApJ, 424, 436

Williams, D. R., Driel-Gesztelyi, L., \& Nakariakov, V. M. 2004, 35th COSPAR Scientific Assembly, 35, 4391

Zhao, J. W., \& Kosovichev, A. G. 2003, ApJ, 591, 446

Zirin, H., \& Tanaka, K. 1973, Sol. Phys., 32, 173 